\title{
Sex Influence on Cardiovascular Autonomic Modulation and on Cardiac and Renal Oxidative Stress: Effects of Aerobic Exercise Training
}

\section{Guilherme Lemos Shimojo}

UNESA São Paulo: Universidade Estacio de Sa - Centro Universitario Estacio Radial de Sao Paulo

Filipe Stoyell-Conti

Universidade Nove de Julho

\section{Danielle Dias}

Universidade Nove de Julho

\section{Catarina Barboza}

Sao Judas Tadeu University: Universidade Sao Judas Tadeu

\section{Michelle Sartori}

Sao Judas Tadeu University: Universidade Sao Judas Tadeu

\section{Nathalia Bernardes}

Universidade Nove de Julho

Iris Sanches

Sao Judas Tadeu University: Universidade Sao Judas Tadeu

\section{Maria-Cláudia Irigoyen}

University of Sao Paulo: Universidade de Sao Paulo

KATIA DE ANGELIS ( $\square$ prof.kangelis@yahoo.com.br)

Universidade Federal de São Paulo, UNIFESP https://orcid.org/0000-0002-3640-9049

\section{Research}

Keywords: Sex, aerobic exercise training, cardiovascular autonomic modulation, oxidative stress

Posted Date: December 8th, 2020

DOI: https://doi.org/10.21203/rs.3.rs-121766/v1

License: (c) (1) This work is licensed under a Creative Commons Attribution 4.0 International License. Read Full License 


\section{Abstract}

The aim of this study was to investigate the difference between sexes after 8 weeks of aerobic exercise training on cardiovascular autonomic modulation and oxidative stress. Wistar rats were distributed into $(n=8 /$ group): sedentary male (SM), trained male (TM), sedentary female (SF) and trained female (TF). Arterial pressure (AP) signals were directly recorded. Cardiovascular autonomic modulation was evaluated by spectral analysis. Aerobic exercise training was performed on treadmill ( 5 days/wk; 8 wks). Oxidative stress was evaluated on cardiac and renal tissues. Both trained groups presented bradycardia. Exercise training increased heart rate variability (VAR-PI) and decreased cardiac sympathovagal balance in both sexes. Although, no differences observed in AP, the female groups showed a lower vascular sympathetic modulation than the male groups. There was a positive correlation between vascular sympathetic modulation and renal membrane lipid peroxidation (LPO) $(r=0.6, p<0.01)$. LPO in cardiac tissue was reduced in the TM, SF and TF groups when compared to the SM group and it was correlated with cardiac sympathetic modulation $(r=0.6, p<0.01)$. Only the TF group presented AP variability and renal LPO decreased and cardiac glutathione redox balance (GSH/GSSG) improved when compared to the other groups. Moreover, the trained females showed greater improvement on VAR-PI ( 1.8 times), on cardiac and renal antioxidant capacity (TRAP: 2,1 and 1.5 times, respectively), and on cardiac GSSG $(\sim 2.8$ times) than the trained males. In conclusion, female rats showed additional aerobic exercise training induced-cardiovascular autonomic modulation and target organs oxidative stress profile improvement than male rats.

\section{Introduction}

Indeed, in young adults and middle age, there is a higher prevalence of cardiovascular disease (CVD) in men than in women, which suggests that sex-related differences in sex hormones might have a key role in the development CVD [1]. Sex hormones exert metabolic, hemodynamic and cardiac autonomic effects that may explain sex differences in the relevance of risk factors of global cardiovascular risk [1, 2]. Clinical and experimental studies report that cardiovascular autonomic regulation also plays an important role in cardiac mortality [3,4]. Previous studies showed that sex-related differences in cardiac autonomic modulation [5]. Clinical and pre-clinical studies have identified a higher resting heart rate, cardiac vagal tone and lower cardiac sympathetic tonus in women compared to man [6].

In fact, Kuo et al. [7] showed that young women have a higher parasympathetic modulation and a lower sympathetic modulation than men, however, the sex-related difference in parasympathetic regulation diminishes after age 50 years, whereas a significant time delay for the disappearance of sympathetic dominance occurs in men. It coincides with the menopause, when there is a marked reduction on female sex hormones. Together, these findings suggest that estrogen has a protective effect on cardiac autonomic modulation response, while endogenous androgen have a detrimental effect on the development of CVD. 
Oxidative stress is another important mechanism involved in the pathophysiology of a large numbers of diseases such hypertension, stroke and other cardiovascular diseases [8, 9]. Previous studies have suggested a link between sex and oxidative stress [9]. In this sense, Borrás et al. [10] showed that female Wistar rats have higher antioxidant gene expression and lower oxidative stress than males. Additionally, studies from our group have shown that autonomic dysfunctions, such as an increased cardiac sympathetic modulation, has been correlated with increase in markers of oxidative stress $[11,12]$.

On the other hand, regular physical activity promotes numerous beneficial adaptations, as well as reduces the risk of chronic diseases $[13,14]$. It is well known that acute exercise activates the sympathetic drive and generates reactive oxygen species (ROS) $[15,16]$. However, regular (chronic) exercise training improves cardiovascular autonomic control and up-regulates the antioxidants profile in heart and kidney $[17,18]$. In this regard, our group has shown benefits on cardiovascular and autonomic control parameters in healthy females [19] as well as in male rats [20] after a protocol of moderateintensity aerobic exercise training. Nevertheless, the link between cardiac autonomic modulation and oxidative stress under aerobic exercise training stimulus in sex differences are still unclear.

In this study, since that autonomic nervous system has an important role of the management oxidative stress status and that aerobic exercise training would induce benefits in these important mechanisms in both sexes. We tested the hypothesis that healthy females present better cardiovascular autonomic control and oxidative status in target organs than healthy males and are more responsible to exercise training-induced adaptations. To address this issue, we investigated the difference between sexes after 8 weeks of aerobic exercise training on cardiovascular autonomic modulation and oxidative stress in heathy male and female rats.

\section{Material And Methods}

\section{Animals and groups.}

Male and female Wistar rats (200-230 g) were obtained from the Animal Facilities of São Judas Tadeu University (São Paulo, SP, Brazil). The animals received freely available standard laboratory chow and water and were housed in a controlled room temperature $\left(22^{\circ} \mathrm{C}\right)$ and kept under controlled 12 -h light-dark cycle. Were assigned into 4 groups ( $\mathrm{n}=8$ each): sedentary male (SM), trained male (TM), sedentary female (SF) and trained female (TF). All surgical procedures and protocols were approved by the ethics committee of São Judas Tadeu University (Protocol \#076/2004), and were conducted in accordance with the National Guidelines for the Care and Use of Laboratory Animals. For the female groups, all in vivo evaluations and euthanasia were conducted on non-ovulatory phase of estrous cycle.

\section{Aerobic exercise training.}

Aerobic exercise training (AT) was performed on a motor treadmill (Imbramed TK-01, Brazil) at low-tomoderate intensity ( $50-60 \%$ maximum running speed) for 1 h a day, 5 days a week for 8 weeks, with a 
gradual increase in speed from 0.3 to $1.0 \mathrm{~km} / \mathrm{h}$. All animals were adapted to the procedure $(10 \mathrm{~min} /$ day; $0.3 \mathrm{~km} / \mathrm{h}$ ) for 5 days before the beginning of the exercise training protocol. After adaptation, the sedentary group was exposed to exercise only during the maximum treadmill test. In order to provide a similar environment and manipulation, sedentary animals were placed on the stationary treadmill three times a week. Sedentary and trained rats were submitted to a maximum treadmill test as described in detail in a previous study [21].Tests were performed at the beginning of the experiment and in the 4th and 8th weeks of the training protocol. The purpose was to determine physical capacity and exercise training intensity [21].

\section{Cardiovascular measurements.}

On the day following the last exercise session, rats were anaesthetized with an intraperitoneal injection of ketamine $(90 \mathrm{mg} / \mathrm{kg}$ ) and xylazine ( $20 \mathrm{mg} / \mathrm{kg}$ ) to implant 2 polyethylene-tipped Tygon cannulas filled with heparinized saline into the right carotid artery and jugular vein for direct measurements of arterial pressure and drug administration, respectively. The free ends of the cannulas were tunneled subcutaneously and exteriorized at the top of the skull. To avoid detraining, hemodynamic measurements were made in conscious, freely moving rats in their home cage $24 \mathrm{~h}$ after surgery, since at that time no significant differences had been observed in AP values $(7,32)$. The arterial cannula was connected to a transducer (Blood Pressure XDCR, Kent® Scientific, USA), and AP signals were recorded for a 30-min period using a microcomputer equipped with an analog-to-digital converter (Windaq, 2Kz, DATAQ Instruments, USA). The recorded data were analyzed on a beat-to-beat basis to quantify changes in systolic (SAP), diastolic (DAP) and mean AP (MAP) and heart rate (HR).

After basal AP measurements, baroreflex sensitivity was evaluated using increasing doses of phenylephrine ( 0.5 to $2.0 \mu \mathrm{g} / \mathrm{mL}$ ) and sodium nitroprusside ( 5 to $20 \mu \mathrm{g} / \mathrm{mL}$ ), given as sequential bolus injections $(0.1 \mathrm{~mL})$ to produce AP rise and fall responses ranging from 5 to $40 \mathrm{mmHg}$ each. Baroreflex sensitivity was assessed by a mean index relating changes in HR to changes in MAP, allowing a separate analysis of gain for reflex bradycardia and reflex tachycardia as described elsewhere [22-24]. Standard deviation from the mean of three time series of $5 \mathrm{~min}$ for each animal was used to obtain the pulse interval (PI) and SAP variabilities in time-domain. For frequency domain analysis, the same time series of $\mathrm{PI}$ and SAP were cubic spline interpolated $(250 \mathrm{~Hz})$ and cubic spline decimated to be equally spaced in time after linear trend removal; power spectral density was obtained through the Fast Fourier Transformation. Spectral power for low-frequency (LF; $0.20-0.75 \mathrm{~Hz})$ and high-frequency (HF; 0.75$4.0 \mathrm{~Hz}$ ) bands were calculated by power spectrum density integration within each frequency bandwidth, using a customized routine (MATLAB 6.0, Mathworks). The coherence between the PI and SAP signal variability was assessed through cross-spectral analysis [24].

\section{Oxidative stress evaluations.}


After cardiovascular evaluations, the animals were killed by decapitation, the heart (ventricles) and kidney (right) were immediately removed, rinsed in saline, and trimmed to remove fat tissue and visible connective tissue. Tissues were then cut into small pieces, placed in ice-cold buffer, and homogenized in an ultra-Turrax blender with $1 \mathrm{~g}$ of tissue per $5 \mathrm{~mL}$ of $150 \mathrm{mmol} / \mathrm{L} \mathrm{KCl}$ and $20 \mathrm{nmol} / \mathrm{L}$ phosphate buffer, $\mathrm{pH}$ 7.4. Homogenates (6-8 animals/group) were centrifuged at $600 \mathrm{~g}$ for 10 minutes at $2{ }^{\circ} \mathrm{C}$. Protein was determined by the method of Lowry et al. using bovine serum albumin as the standard [25].

\section{Lipid peroxidation.}

Lipid peroxidation (LPO) was measured by the tert-butyl hydroperoxide-initiated chemiluminescence (CL) assay, as previously described by Gonzalez Flecha et al. [26] CL assay was carried out with an LKB Rack Beta liquid scintillation spectrometer 1215 (LKB Producer AB) in the out-of-coincidence mode at room temperature $\left(25^{\circ} \mathrm{C}\right.$ to $27^{\circ} \mathrm{C}$ ). The supernatants were diluted in $140 \mathrm{mmol} / \mathrm{L} \mathrm{KCl}$ and $20 \mathrm{mmol} / \mathrm{L}$ phosphate buffer, $\mathrm{pH} 7.4$, and added to glass tubes, which were placed in scintillation vials; $3 \mathrm{mmol} / \mathrm{L}$ tert-butylhydroperoxide was added, and CL was determined as the maximum level of emission.

Total radical- trapping antioxidant potential. TRAP, which indicates the total antioxidant capacity present in a homogenate was measured by chemiluminescence using 2,2'-azo-bis(2-amidinopropane) (ABAP, a source of alkyl peroxyl free radicals) and luminal. A misture consisting of $20 \mathrm{mmol}$.L-1 luminol, and 50 mmol..$^{-1}$ phosphate buffer $(\mathrm{pH}=7.4)$ was incubated to achieve a steady-state luminescente from the free radical-mediated luminal oxidation. A calibration curve was obtained by using different concentrations (between 0.2 and $1 \mu \mathrm{mol} . \mathrm{L}^{-1}$ ) of Trolox (hydrosoluble vitamim E) [27]. Luminescence was measured in a liquid scintillation counter using the out-of-coincidence mode.

\section{Antioxidant enzyme.}

Superoxide dismutase activity (SOD) was measured spectrophotometrically in heart homogenates by rate inhibition of pyrogallol autooxidation at $420 \mathrm{~nm}$ (22). Catalase activity (CAT) was measured by monitoring the decrease in $\mathrm{H}_{2} \mathrm{O}_{2}$ concentration at $240 \mathrm{~nm}$ [28]. Glutathione peroxidase activity (GPx) was determined by monitoring NADPH oxidation spectrophotometrically at $340 \mathrm{~nm}$ [29].

Determination of oxidized and reduced glutathione concentration. To determine oxidized and reduced glutathione concentration, only cardiac tissue was deproteinized with $2 \mathrm{~mol} / \mathrm{l}$ perchloric acid, centrifuged for $10 \mathrm{~min}$ at $1000 \mathrm{~g}$, and the supernatant was neutralized with $2 \mathrm{~mol} / \mathrm{l}$ potassium hydroxide. The reaction medium contained $100 \mathrm{mmol} / \mathrm{l}$ phosphate buffer $(\mathrm{pH} 7.2), 2 \mathrm{mmol} / \mathrm{l}$ nicotinamide dinucleotide phosphate acid, $0.2 \mathrm{U} / \mathrm{ml}$ glutathione reductase, and $70 \mathrm{mmol} / \mathrm{l} 5,50$ dithiobis (2-nitrobenzoic acid). To determine reduced glutathione, the supernatant was neutralized with $2 \mathrm{~mol} / \mathrm{l}$ potassium hydroxide, to react with $70 \mathrm{mmol} / \mathrm{I}$ 5,50 dithiobis (2-nitro benzoic acid), and the absorbance values measured at $420 \mathrm{~nm}[29]$. 


\section{Statistical analysis}

Data are presented as mean \pm SEM. Levene's test was used to assess variance homogeneity. Analysis of variance (ANOVA) was used to compare the 4 groups, followed by Student Newman Keuls post hoc test. Pearson correlation was used to study the association between variables ( $n=6-8$ animals/group). The significance level was established at $p<0.05$.

\section{Results}

\section{Metabolic evaluations.}

In the beginning and at the end of the protocol, the male groups showed a higher body weight (BW) than the female groups (SM: $285 \pm 4$ and TM: $297 \pm 2$ vs SF: $215 \pm 5$ and TF: $207 \pm 5$ grams). The aerobic exercise was not able to attenuate the increase of the body weight throughout the protocol (Delta BW SM: $96.8 \pm 4.7$ vs TM: $86.2 \pm 6.7$ and SF: $49.4 \pm 2.7$ vs TF: $40.0 \pm 4$ grams).

\section{Maximal exercise capacity.}

At the beginning of the experiment, maximal exercise capacity was similar among the studied groups (SM: $1.75 \pm 0.03$, TM: $1.71 \pm 0.04$, SF: $1.83 \pm 0.06$ and TF: $1.86 \pm 0.17 \mathrm{~km} / \mathrm{h}$ ). However, after 8 weeks of exercise training, the trained animals (TM and TF) demonstrated an increase in maximal speed of running compared with sedentary animals (TM: $2.2 \pm 0.06, \mathrm{TF}: 2.4 \pm 0.11$ vs. SM: $1.71 \pm 0.10$ and SF $1.8 \pm$ $0.15 \mathrm{~km} / \mathrm{h}$ ). There were no significant differences between sexes.

\section{Cardiovascular measurements.}

Hemodynamic data are described on Table 1. Both sedentary groups exhibited similar values in arterial pressure. Exercise training did not change AP (systolic, diastolic and mean) in both sexes. However, trained groups exhibited reduced HR when compared to sedentary ones. 
Table 1

Hemodynamic and cardiac autonomic control in sedentary male (SM), trained male (TM), sedentary female (SF) and trained female (TF).

\begin{tabular}{|lllll|}
\hline Measurement & SM & TM & SF & TF \\
\hline MAP $(\mathrm{mmHg})$ & $117 \pm 2$ & $116 \pm 2$ & $113 \pm 2$ & $110 \pm 3$ \\
\hline DAP $(\mathrm{mmHg})$ & $100 \pm 2$ & $99 \pm 3$ & $95 \pm 2$ & $96 \pm 2$ \\
\hline SAP $(\mathrm{mmHg})$ & $134 \pm 2$ & $135 \pm 2$ & $128 \pm 2$ & $131 \pm 2$ \\
\hline HR (bpm) & $351 \pm 6$ & $331 \pm 4 \dagger$ & $357 \pm 6$ & $334 \pm 7 \dagger$ \\
\hline
\end{tabular}

Data are reported as mean \pm SEM. $\dagger P<0.05$ vs. SF. MAP: mean arterial pressure; SAP: systolic arterial pressure; DAP: diastolic arterial pressure; HR: heart rate.

Table 2

Cardiac and renal antioxidant enzyme activity in sedentary male (SM), trained male (TM), sedentary female (SF) and trained female (TF).

\begin{tabular}{|lllll|}
\hline Measurement & SM & TM & SF & TF \\
\hline Cardiac & & & & \\
\hline CAT (pmol/mg protein) & $0.5 \pm 0.02$ & $0.8 \pm 0.04^{*}$ & $0.8 \pm 0.04^{*}$ & $0.7 \pm 0.05^{*}$ \\
\hline SOD (U/mg protein) & $15 \pm 0.64$ & $14 \pm 0.78$ & $16 \pm 0.58$ & $16 \pm 0.37$ \\
\hline GPx ( $\mu$ mol/mg protein) & $27 \pm 0.73$ & $11 \pm 0.64^{*}$ & $32 \pm 3.55 \#$ & $35 \pm 1.57^{*} \#$ \\
\hline Renal & & & & \\
\hline CAT (pmol/mg protein) & $1.97 \pm 0.19$ & $4.15 \pm 0.17^{*}+$ & $1.72 \pm 0.16$ & $2.44 \pm 0.43^{*} \#$ \\
\hline SOD (U/mg protein) & $10.8 \pm 0.54$ & $8.24 \pm 1.08$ & $9.48 \pm 0.52$ & $10.97 \pm 1.18$ \\
\hline GPx ( $\mu$ mol/mg protein) & $60.7 \pm 5.22$ & $36.7 \pm 3.36^{*}$ & $43.0 \pm 3.0 *$ & $33.6 \pm 5.33^{*}$ \\
\hline $\begin{array}{l}\text { Data are reported as mean } \pm \text { SEM. * } P<0.05 \text { vs. SM; \# } P<0.05 \text { vs. TM; }+P<0.05 \text { vs. SF. CL: } \\
\text { chemiluminescence; CAT: catalase; SOD: Superoxide dismutase; GPx: Glutathione peroxidase). }\end{array}$ \\
\hline
\end{tabular}

\section{Cardiovascular autonomic evaluations.}

Regarding the autonomic modulation evaluation in time and frequency domains, there was an increase in total variance of PI (VAR-PI) in the trained groups when compared to the sedentary groups (TM: $69.2 \pm 7.2$ and TF: $76.2 \pm 7.6$ vs. SM: $49.5 \pm 5.8$; SF: $44.5 \pm 5.7 \mathrm{~ms}^{2}$ ) (Fig. 1a). There was no difference in RMSSD between groups. The LF band of PI was reduced in both trained groups (TM:19.3 \pm 1.0 and TF: $20.9 \pm 1.6$ vs. SM: $26.3 \pm 1.4$; SF: $27.5 \pm 2.1 \mathrm{nu}$ ), while the HF band of the PI was increased in both trained groups when compared to their respectively sedentary groups (TM:80.7 \pm 1.0 and TF: $79.1 \pm 1.6$ vs. SM: $73.7 \pm$ $1.4 ; \mathrm{SF}: 72.5 \pm 2.1 \mathrm{nu}$ ). Additionally, the trained group showed decreased LF/HF ratio when compared with 
the sedentary groups (Fig. 1b). There was no difference observed in VAR-SAP between groups (Fig. 1c). However, the LF band of SAP in both female groups was lower when compared to the male groups (SF: $3.9 \pm 0.4$; TF: $4.5 \pm 0.6$ vs. SM: $7.3 \pm 0.6$ and TM: $6.9 \mathrm{mmHg}^{2}$ ) (Fig. 1d). Correlations were obtained between SAP and LF band of SAP ( $r=0.7, p<0.001)$ (Fig. 4a), as well as between heart rate and VAR-PI $(r=-0.6, p<0.001)$ and LF/HF ratio $(r=0.7, p<0.001)$ (Fig. 4b).

\section{Cardiac oxidative stress.}

Membrane lipid peroxidation (LPO) as assessed by $\mathrm{CL}$, showed significant reduction in the TM, SF and TF groups (TM: $3442 \pm 172$; SF: $2661 \pm 358$ and TF: $3228 \pm 363 \mu \mathrm{mol} / \mathrm{mg}$ protein) when compared to the SM group (SM: $7323 \pm 457 \mu \mathrm{mol} / \mathrm{mg}$ protein) (Fig. $2 a$ ). CAT activity showed higher values in the TM, SF and TF groups when compared to the SM group (Table 2). GPx activity exhibited similar values in either sedentary groups. After aerobic exercise training, GPx activity showed a significant increase in the TF group when compared to the TM group (Table 2). There was no significant difference observed in SOD activity between studied groups (Table 2). The SF group showed decrease in GSSG when compared to the SM group. However, both trained groups showed lower levels of GSSG when compared with their respective sedentary groups. An additional decrease was observed in TF group when compared to TM group (Fig. 3a). We did not observe differences between groups at GSH levels (Fig. 3b) Aerobic exercise training increased the GSH/GSSG in females (FT group) when compared to other groups (Fig. 3c). The training in males (TM group) had a significant influence on TRAP levels when compared with sedentary groups (TM: $17.6 \pm 2.31$ vs. SM: $7.6 \pm 1.57$ and SF: $7.4 \pm 1.19 \mu \mathrm{M} /$ trolox). Additionally, trained females showed additional increase on TRAP levels when compared to trained males (TF: $28.4 \pm 3.24 \mu \mathrm{M} /$ trolox) (Fig. 2b). A positive correlation was observed between LF band of PI and cardiac LPO $(r=0.6, p<0.01)$ (Fig. 4c).

\section{Renal oxidative stress.}

Membrane lipid peroxidation (LPO) as assessed by $\mathrm{CL}$, showed significant reduction in trained females when compared to the other groups (TF: $1831 \pm 170$ vs. SM: $4825 \pm 395$; TM: $4415 \pm 350$ and SF: $4320 \pm$ $358 \mu \mathrm{mol} / \mathrm{mg}$ protein) (Fig. 2c). CAT activity showed higher values in the TF group when compared to the SM group. Nevertheless, the TM group showed higher CAT activity when compared to the other groups (Table 2). There was no significant difference observed in SOD activity (Table 2). GPx activity showed significant reduction in the TM, SF and TF groups when compared to the SM group (Table 2). The training in males (TM group) had a significant influence on TRAP levels when compared with sedentary groups (TM: $102 \pm 15$ vs. SM: $42.84 \pm 8.29$ and SF: $64.74 \pm 5.78 \mu \mathrm{M} /$ trolox). Additionally, the trained female group showed additional increase on TRAP levels when compared to the trained male group (TF: $196 \pm 18$ $\mu \mathrm{M}$ /trolox) (Fig. 2d). Positive correlations were obtained between renal LPO and SAP $(r=0.6, p<0.01)$ and LF band of SAP $(r=0.6, p<0.01)$ (Fig. 4d). 


\section{Discussion}

In the present study we investigated the difference between sexes on cardiovascular autonomic modulation and oxidative stress in target organs after eight weeks of aerobic exercise training. The major finding of this study is that, besides both trained groups showing improvement on cardiovascular autonomic control and cardiac and renal oxidative stress when compared to the sedentary groups, the female group was more responsive to the exercise training protocol than the male group. This suggests that the sexes may respond differently to aerobic exercise training regarding cardiovascular autonomic control and oxidative stress.

The autonomic nervous system is a key regulator of cardiovascular homeostasis [5]. There are multiple ways to measure cardiac autonomic control; in this study, we used the heart rate and arterial pressure variability, which allows us to assess the role of autonomic nervous system fluctuations in normal healthy individuals and in patients with various cardiovascular and non-cardiovascular disorders [30]. Recent data support that estrogen provides a degree of cardiovascular protection by influence of the autonomic nervous system [5, 31]. In this sense, Kuo et al. [7] showed that healthy women had lower cardiac sympathetic modulation when compared to men before post menopause stage. That result is also in concordance with the lower LF band of SAP observed in the female groups in the present study. These results could explain the lower cardiovascular risk between pre-menopausal women compared to men. Additionally, the aerobic exercise training was able to improve HR variability and simpathovagal balance (LF/HF) independent of sex, which can be explained by a decrease of the LF band of PI and an increase in HF band of PI. In fact, the cardiac benefits induced by aerobic exercise training seems to be not dependent of sex, even with difference on cardiovascular autonomic modulation in sedentary condition [31].

Moreover, studies have shown that dynamic aerobic exercise training does not change arterial blood pressure in male [32] and female [19] normotensive rats. Indeed, in the present study, the AP values obtained did not change after aerobic exercise training in both sexes; however, the female animals showed lower vascular sympathetic modulation than males, which may explain a reduced vasoconstriction and a lower vascular resistance in females in comparison to males, especially after exercise [33]. Interesting, we observed a positive correlation between LF band of SAP and SAP, showing that, despite similar basal values of AP between sexes and condition of training, animals with lower sympathetic vascular modulation presented reduced values of SAP. In the present study, we observed resting bradycardia in trained male and female rats compared to sedentary groups, which can be related with an important reduction in cardiac sympathovagal balance. In fact, supporting this hypothesis we obtained significant correlations involving all groups between HR and VAR-PI ( $r=-0.6)$ and LF/HF ratio $(r=$ 0.7), demonstrating that animals with higher cardiac total variance of $\mathrm{PI}$ or lower cardiac sympathovagal balance showed reduced basal HR.

Previous studies also have shown that female rats run more than male rats [34]. In the present study, 8 weeks of the aerobic exercise training induced an increase of exercise capacity, as evaluated by the 
response to the maximal exercise test, in both sexes. This result corroborates with previous studies in males and females Wistar [34] and ovarectomized rats [11].

Reactive oxygen species (ROS) are formed under both physiological and pathological conditions and have been implicated of a large number of diseases [8,9]. Some studies have shown an association with a decreased sympathetic modulation and an enhanced oxidative stress central and peripherally $[11,12]$. Moreover, accumulating evidences indicate a protective role of estrogen on cardiovascular disorder [22, 35]. In the present study, we observed that sedentary female rats showed less membrane lipid peroxidation in cardiac tissue as assessed by $\mathrm{CL}$ when compared to sedentary male rats. Indeed, estrogen seems to act centrally reducing BP, sympathoexcitation and oxidative stress [36]. Some studies have reported an important role of sex difference in antioxidant capacity and exercise-induced reactive oxygen species production [34,37]. On the other hand, some studies claim that sex does not affect oxidative stress parameters after exercise training [38]. Therefore, the impact of exercise training on oxidative stress between sexes remains contradictory.

We observed that exercise training induced a decrease in both trained groups on membrane lipid peroxidation in cardiac tissue; however, only the trained female group showed a reduced membrane lipid peroxidation in renal tissue. This data reinforce results showing that the response to oxidative stress induced by exercise training is dependent of tissue [39]. However, this response occurred probably due an increase in the antioxidant enzymes activity, such as CAT and GPx, which may be associated with a marked increase of nonenzymatic antioxidant capacity, evidenced by TRAP. In agreement with the present findings, previous data of our group showed a reduction on cardiac oxidative stress markers after aerobic exercise training in female ovariectomized [11] and male hypertensive rats [40]. Glutathione redox balance (GSH/GSSG) in cardiac tissue also was improved in the trained female group in the present study. It is important to note that this nonenzymatic endogenous antioxidant has a major role against oxidative stress [41]. Thus, the better antioxidative profile in trained females in both cardiac and renal tissues may be explained by female sex hormones, which can induce antioxidant enzyme expression by stimulating the antioxidant defense system [42], inhibit the formation of lipid peroxidase in some tissues [43] and lead to a higher mitochondrial/systemic antioxidant capacity [10].

It is important to remain that unfavorable changes in BP or HR variability were correlated with end organ damage in humans and rats, despite normal values of AP [44-46]. Furthermore, it well known that oxidative stress is one of the key mechanisms involved in end organ damage that can be developed during life spam [8]. In this sense, our data provide evidence that changes in cardiovascular autonomic modulation related to sex or condition of training can impact in oxidative stress markers in target organ. We observed significant positive correlations between LF band of PI and cardiac LPO, as well as, between SAP or LF band of SAP with renal LPO. Together, these results suggest that animals with lower cardiac/vascular sympathetic modulation or SAP presented reduced levels of cardiac/renal oxidative damage. 
Indeed, in this study, we showed that there are some differences in autonomic modulation and oxidative stress markers between males and females. Interestingly, female rats seem to be more responsive to a moderate intensity aerobic exercise training. When we compared the sedentary and trained groups, we observed that the TF group showed an increase of $71 \%$ in VAR-PI and $272 \%$ in cardiac and $206 \%$ in renal TRAP, as well as a reduction of $50 \%$ in cardiac GSSG, while the TM group showed an increase of $39 \%$ in VAR-PI and $131 \%$ in cardiac and $142 \%$ in renal TRAP, as well as a reduction of $18 \%$ in cardiac GSSG. Thus, the trained female animals showed greater improvement on VAR-PI ( 1.8 times), on cardiac and renal TRAP $(\sim 2,1$ and $\sim 1.5$ times, respectively $)$ and on cardiac GSSG $(\sim 2.8$ times $)$ than trained male animals. Moreover, despite trained males reduced cardiac LPO to similar levels observed in trained females, only females rat reduced VAR-SAP and renal LPO and improved cardiac glutathione redox balance in response to aerobic exercise training.

In conclusion, aerobic exercise training protocol improved cardiovascular autonomic modulation and oxidative stress profile in target organs in both sexes. The favorable changes on BP and HR variability were associated with hemodynamic improvement and reduced end organ damage. The exercise training benefits were more pronounced in females than males. Therefore, findings from the current study suggest that sex was an important determinant of changes induced by aerobic exercise training. Our data reinforce that sex should be considered to understand autonomic modulation and oxidative stressinduced cardiovascular dysfunction, as well as, the effects of different approach to prevent/treat cardiovascular disease.

\section{Perspectives and Significance}

There is a higher prevalence of cardiovascular disease in men than in women, which suggests that sexrelated differences in sex hormones might have a key role in the development CVD. Additionally, regular physical activity promotes numerous beneficial adaptations, as well as reduces the risk of chronic diseases. Our findings contribute to a fuller understanding of the impact of aerobic exercise training on cardiovascular autonomic control and oxidative stress between gender. In this since, we observed in an experimental model that female rats showed additional aerobic exercise training induced-cardiovascular autonomic modulation and target organs oxidative stress profile improvement than male rats. As the estimates of cardiovascular mortality in the coming years are still quite challenging, our data reinforce the need for further research on the effects of regular exercise on the mechanisms of autonomic cardiovascular control, oxidative stress and immunity between genders.

\section{Declarations}

\section{Acknowledgments}

Not applicable

\section{Funding}


This study was supported by grants from Fundação de Amparo e Pesquisa do Estado de São Paulo (FAPESP: 2007/57595-5; 2018/17183-4) and Conselho Nacional de Desenvolvimento Científico e Tecnológico (CNPq). MCl and KDA are recipients of CNPq-BPQ fellowships.

\section{Author contributions}

G.L.S, M.C.I., and K.D.A. conception and design of research; G.L.S., F.F.S-C., D.S.D., C.B., N.B., and I.C.S, performed experiments; G.L.S., F.F.S-C., D.S.D., C.B., N.B., I.C.S., and K.D.A. analyzed data; G.L.S., F.F.S-C and K.D.A. interpreted results of experiments; G.L.S and F.F.S-C. prepared figures; G.L.S., F.F.S-C and K.D.A. drafted manuscript; G.L.S., F.F.S-C., M.C.I., and K.D.A. edited and revised manuscript; G.L.S., F.F.S-C., D.S.D., C.B., N.B., I.C.S, M.C.I., and K.D.A. approved final version of manuscript.

\section{Availability of data and materials}

All data are available from the corresponding author upon request.

\section{Ethics approval and consent to participate}

The research was approved by the ethics committee of Nove de Julho university.

\section{Consent for publication}

Not applicable.

\section{Competing interests}

The author declare that they have no competing interests.

\section{References}

1. Vitale C, Mendelsohn ME, Rosano GM. Gender differences in the cardiovascular effect of sex hormones. Nat Rev Cardiol. 2009;6:8:532-42; doi:10.1038/nrcardio.2009.105.

2. Dart AM, Du XJ, Kingwell BA. Gender, sex hormones and autonomic nervous control of the cardiovascular system. Cardiovasc Res. 2002;53:3:678-87.

3. Kleiger RE, Miller JP, Bigger JT, Jr., Moss AJ. Decreased heart rate variability and its association with increased mortality after acute myocardial infarction. Am J Cardiol. 1987;59:4:256-62.

4. Schwartz PJ, Billman GE, Stone HL. Autonomic mechanisms in ventricular fibrillation induced by myocardial ischemia during exercise in dogs with healed myocardial infarction. An experimental 
preparation for sudden cardiac death. Circulation. 1984;69:4:790-800.

5. Pothineni NV, Shirazi LF, Mehta JL. Gender Differences in Autonomic Control of the Cardiovascular System. Current pharmaceutical design. 2016;22:25:3829-34.

6. Joyner MJ, Barnes JN, Hart EC, Wallin BG, Charkoudian N. Neural control of the circulation: how sex and age differences interact in humans. Compr Physiol. 2015;5:1:193-215; doi:10.1002/cphy.c140005.

7. Kuo TB, Lin T, Yang CC, Li CL, Chen CF, Chou P. Effect of aging on gender differences in neural control of heart rate. The American journal of physiology. 1999;277:6:H2233-9; doi:10.1152/ajpheart.1999.277.6.H2233.

8. Liguori I, Russo G, Curcio F, Bulli G, Aran L, Della-Morte D, et al. Oxidative stress, aging, and diseases. Clinical interventions in aging. 2018;13:757.

9. Kander MC, Cui Y, Liu Z. Gender difference in oxidative stress: a new look at the mechanisms for cardiovascular diseases. Journal of cellular and molecular medicine. 2017;21:5:1024-32.

10. Borrás C, Sastre J, García-Sala D, Lloret A, Pallardó FV, Viña J. Mitochondria from females exhibit higher antioxidant gene expression and lower oxidative damage than males. Free radical biology and medicine. 2003;34:5:546-52.

11. Irigoyen MC, Paulini J, Flores LJ, Flues K, Bertagnolli M, Moreira ED, et al. Exercise training improves baroreflex sensitivity associated with oxidative stress reduction in ovariectomized rats. Hypertension (Dallas, Tex : 1979). 2005;46:4:998-1003; doi:10.1161/01.HYP.0000176238.90688.6b.

12. Bertagnolli M, Campos C, Schenkel PC, de Oliveira VL, De Angelis K, Bello-Klein A, et al. Baroreflex sensitivity improvement is associated with decreased oxidative stress in trained spontaneously hypertensive rat. Journal of hypertension. 2006;24:12:2437-43;

doi:10.1097/01.hjh.0000251905.08547.17.

13. Fiuza-Luces C, Garatachea N, Berger NA, Lucia A. Exercise is the real polypill. Physiology. 2013;28:5:330-58.

14. Pedersen BK, Saltin B. Exercise as medicine - evidence for prescribing exercise as therapy in 26 different chronic diseases. Scandinavian journal of medicine \& science in sports. 2015;25 Suppl 3:172; doi:10.1111/sms.12581.

15. Powers SK, Nelson WB, Hudson MB. Exercise-induced oxidative stress in humans: cause and consequences. Free Radical Biology and Medicine. 2011;51:5:942-50.

16. Christensen NJ, Galbo H. Sympathetic nervous activity during exercise. Annual review of physiology. 1983;45:139-53; doi:10.1146/annurev.ph.45.030183.001035.

17. Shimojo GL, da Silva Dias D, Malfitano C, Sanches IC, Llesuy S, Ulloa L, et al. Combined Aerobic and Resistance Exercise Training Improve Hypertension Associated With Menopause. Frontiers in physiology. 2018;9:1471; doi:10.3389/fphys.2018.01471.

18. Bayod S, Del Valle J, Lalanza JF, Sanchez-Roige S, de Luxan-Delgado B, Coto-Montes A, et al. Longterm physical exercise induces changes in sirtuin 1 pathway and oxidative parameters in adult rat tissues. Experimental gerontology. 2012;47:12:925-35; doi:10.1016/j.exger.2012.08.004. 
19. Sanches IC, Sartori M, Jorge L, Irigoyen MC, De Angelis K. Tonic and reflex cardiovascular autonomic control in trained-female rats. Brazilian journal of medical and biological research $=$ Revista brasileira de pesquisas medicas e biologicas. 2009;42:10:942-8.

20. Farah D, Nunes J, Sartori M, Dias DD, Sirvente R, Silva MB, et al. Exercise Training Prevents Cardiovascular Derangements Induced by Fructose Overload in Developing Rats. PloS one. 2016;11:12:e0167291; doi:10.1371/journal.pone.0167291.

21. Rodrigues B, Figueroa DM, Mostarda CT, Heeren MV, Irigoyen MC, De Angelis K. Maximal exercise test is a useful method for physical capacity and oxygen consumption determination in streptozotocindiabetic rats. Cardiovascular diabetology. 2007;6:38; doi:10.1186/1475-2840-6-38.

22. Flues K, Paulini J, Brito S, Sanches IC, Consolim-Colombo F, Irigoyen MC, et al. Exercise training associated with estrogen therapy induced cardiovascular benefits after ovarian hormones deprivation. Maturitas. 2010;65:3:267-71; doi:10.1016/j.maturitas.2009.11.007.

23. Sanches IC, de Oliveira Brito J, Candido GO, da Silva Dias D, Jorge L, Irigoyen MC, et al. Cardiometabolic benefits of exercise training in an experimental model of metabolic syndrome and menopause. Menopause (New York, NY). 2012;19:5:562-8; doi:10.1097/gme.0b013e3182358c9c.

24. Bernardes N, da Silva Dias D, Stoyell-Conti FF, de Oliveira Brito-Monzani J, Malfitano C, Caldini EG, et al. Baroreflex Impairment Precedes Cardiometabolic Dysfunction in an Experimental Model of Metabolic Syndrome: Role of Inflammation and Oxidative Stress. Scientific reports. 2018;8:1:8578; doi:10.1038/s41598-018-26816-4.

25. Lowry OH, Rosebrough NJ, Farr AL, Randall RJ. Protein measurement with the Folin phenol reagent. The Journal of biological chemistry. 1951;193:1:265-75.

26. Gonzalez Flecha B, Llesuy S, Boveris A. Hydroperoxide-initiated chemiluminescence: an assay for oxidative stress in biopsies of heart, liver, and muscle. Free radical biology \& medicine. 1991;10:2:93100.

27. Evelson P, Travacio M, Repetto M, Escobar J, Llesuy S, Lissi EA. Evaluation of total reactive antioxidant potential (TRAP) of tissue homogenates and their cytosols. Archives of biochemistry and biophysics. 2001;388:2:261-6; doi:10.1006/abbi.2001.2292.

28. Aebi H. Catalase in vitro. Methods in enzymology. 1984;105:121-6.

29. Flohe L, Gunzler WA. Assays of glutathione peroxidase. Methods in enzymology. 1984;105:114-21.

30. Pagani M. [Sympathetic-vagal euqilibrium and physical exercise]. Cardiologia (Rome, Italy). 1997;42:7:669-80.

31. Dutra SG, Pereira AP, Tezini GC, Mazon JH, Martins-Pinge MC, Souza HC. Cardiac autonomic modulation is determined by gender and is independent of aerobic physical capacity in healthy subjects. PloS one. 2013;8:10:e77092; doi:10.1371/journal.pone.0077092.

32. De Angelis KL, Oliveira AR, Werner A, Bock P, Bello-Klein A, Fernandes TG, et al. Exercise training in aging: hemodynamic, metabolic, and oxidative stress evaluations. Hypertension (Dallas, Tex : 1979). 1997;30:3 Pt 2:767-71. 
33. Bassareo P, Crisafulli A. Gender differences in hemodynamic regulation and cardiovascular adaptations to dynamic exercise. Current cardiology reviews. 2019.

34. Yamamoto T, Ohkuwa T, Itoh H, Sato Y, Naoi M. Effect of gender differences and voluntary exercise on antioxidant capacity in rats. Comparative biochemistry and physiology Toxicology \& pharmacology : CBP. 2002;132:4:437-44.

35. Iorga A, Cunningham CM, Moazeni S, Ruffenach G, Umar S, Eghbali M. The protective role of estrogen and estrogen receptors in cardiovascular disease and the controversial use of estrogen therapy. Biology of sex differences. 2017;8:1:33; doi:10.1186/s13293-017-0152-8.

36. Hao F, Gu Y, Tan X, Deng Y, Wu ZT, Xu MJ, et al. Estrogen Replacement Reduces Oxidative Stress in the Rostral Ventrolateral Medulla of Ovariectomized Rats. Oxidative medicine and cellular longevity. 2016;2016:2158971; doi:10.1155/2016/2158971.

37. Goldfarb AH, McKenzie MJ, Bloomer RJ. Gender comparisons of exercise-induced oxidative stress: influence of antioxidant supplementation. Applied physiology, nutrition, and metabolism = Physiologie appliquee, nutrition et metabolisme. 2007;32:6:1124-31; doi:10.1139/h07-078.

38. Pepe H, Balci SS, Revan S, Akalin PP, Kurtoglu F. Comparison of oxidative stress and antioxidant capacity before and after running exercises in both sexes. Gender medicine. 2009;6:4:587-95; doi:10.1016/j.genm.2009.10.001.

39. Balci SS, Pepe H. Effects of gender, endurance training and acute exhaustive exercise on oxidative stress in the heart and skeletal muscle of the rat. The Chinese journal of physiology. 2012;55:4:23644; doi:10.4077/cjp.2012.baa021.

40. Bertagnolli M, Schenkel PC, Campos C, Mostarda CT, Casarini DE, Bello-Klein A, et al. Exercise training reduces sympathetic modulation on cardiovascular system and cardiac oxidative stress in spontaneously hypertensive rats. American journal of hypertension. 2008;21:11:1188-93; doi:10.1038/ajh.2008.270.

41. Hanschmann EM, Godoy JR, Berndt C, Hudemann C, Lillig CH. Thioredoxins, glutaredoxins, and peroxiredoxins-molecular mechanisms and health significance: from cofactors to antioxidants to redox signaling. Antioxidants \& redox signaling. 2013;19:13:1539-605; doi:10.1089/ars.2012.4599.

42. Katalinic V, Modun D, Music I, Boban M. Gender differences in antioxidant capacity of rat tissues determined by 2,2'-azinobis (3-ethylbenzothiazoline 6-sulfonate; ABTS) and ferric reducing antioxidant power (FRAP) assays. Comparative biochemistry and physiology Toxicology \& pharmacology : CBP. 2005;140:1:47-52; doi:10.1016/j.cca.2005.01.005.

43. Huh K, Shin US, Choi JW, Lee SI. Effect of sex hormones on lipid peroxidation in rat liver. Archives of pharmacal research. 1994;17:2:109-14.

44. Heart rate variability: standards of measurement, physiological interpretation and clinical use. Task Force of the European Society of Cardiology and the North American Society of Pacing and Electrophysiology. Circulation. 1996;93:5:1043-65.

45. Flues K, Moraes-Silva I, Mostarda C, Souza P, Diniz G, Moreira E, et al. Cardiac and pulmonary arterial remodeling after sinoaortic denervation in normotensive rats. Autonomic Neuroscience. 2012;166:1- 
2:47-53.

46. Irigoyen M-C, De Angelis K, Dos Santos F, Dartora DR, Rodrigues B, Consolim-Colombo FM.

Hypertension, blood pressure variability, and target organ lesion. Current hypertension reports. 2016;18:4:31.

Figures

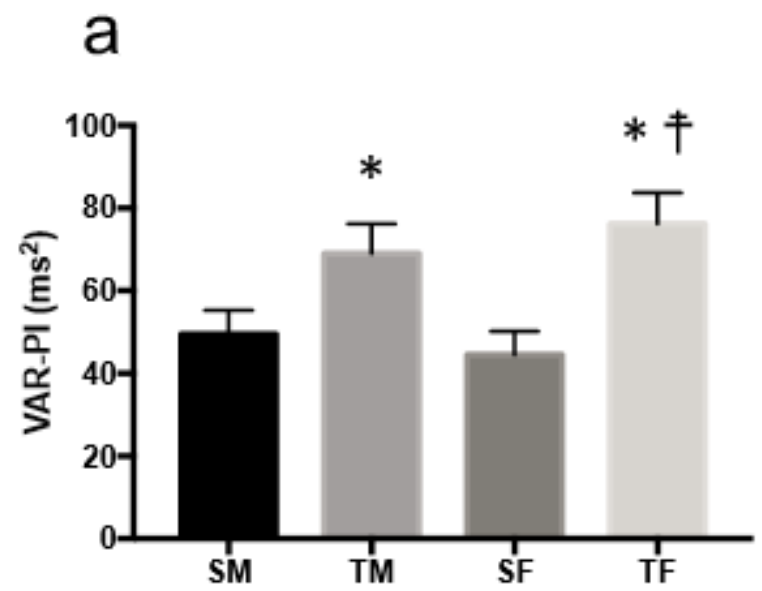

b

C

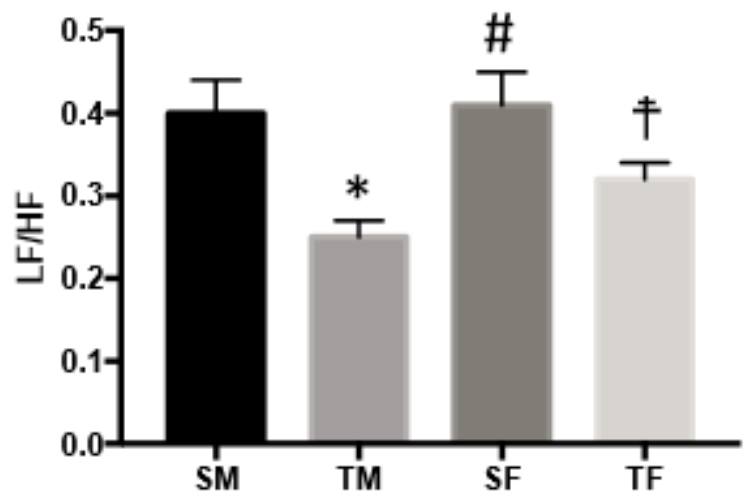

d
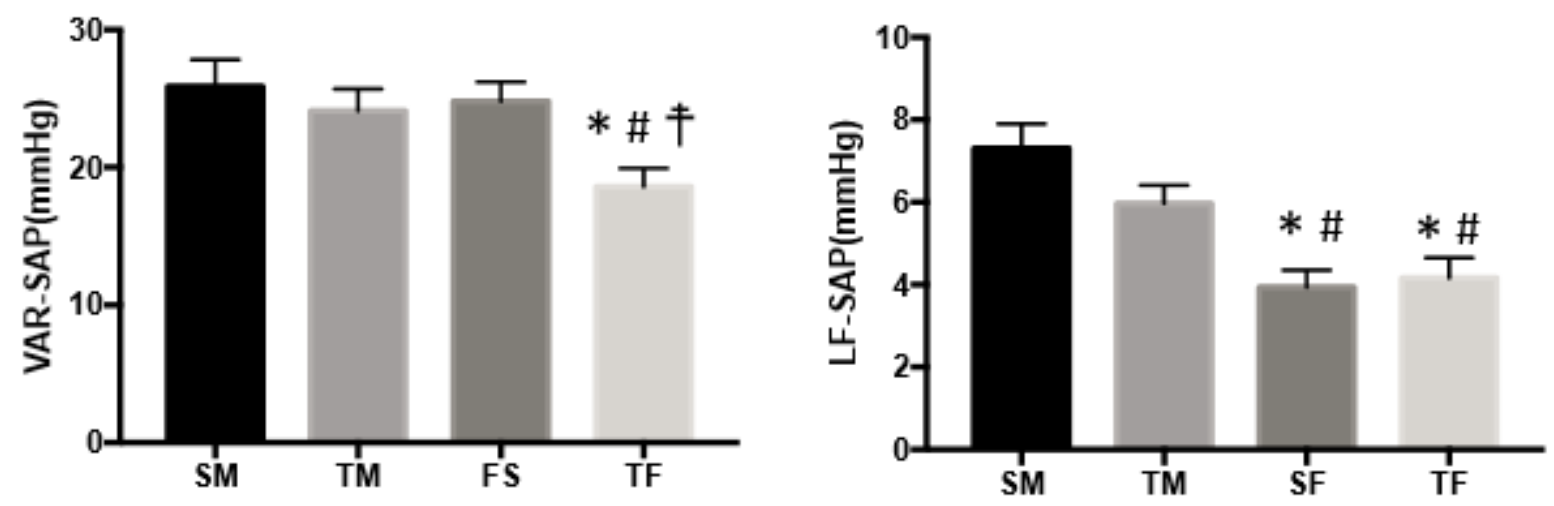

Figure 1

Female showed lower Sympathetic modulation in experimental model. 

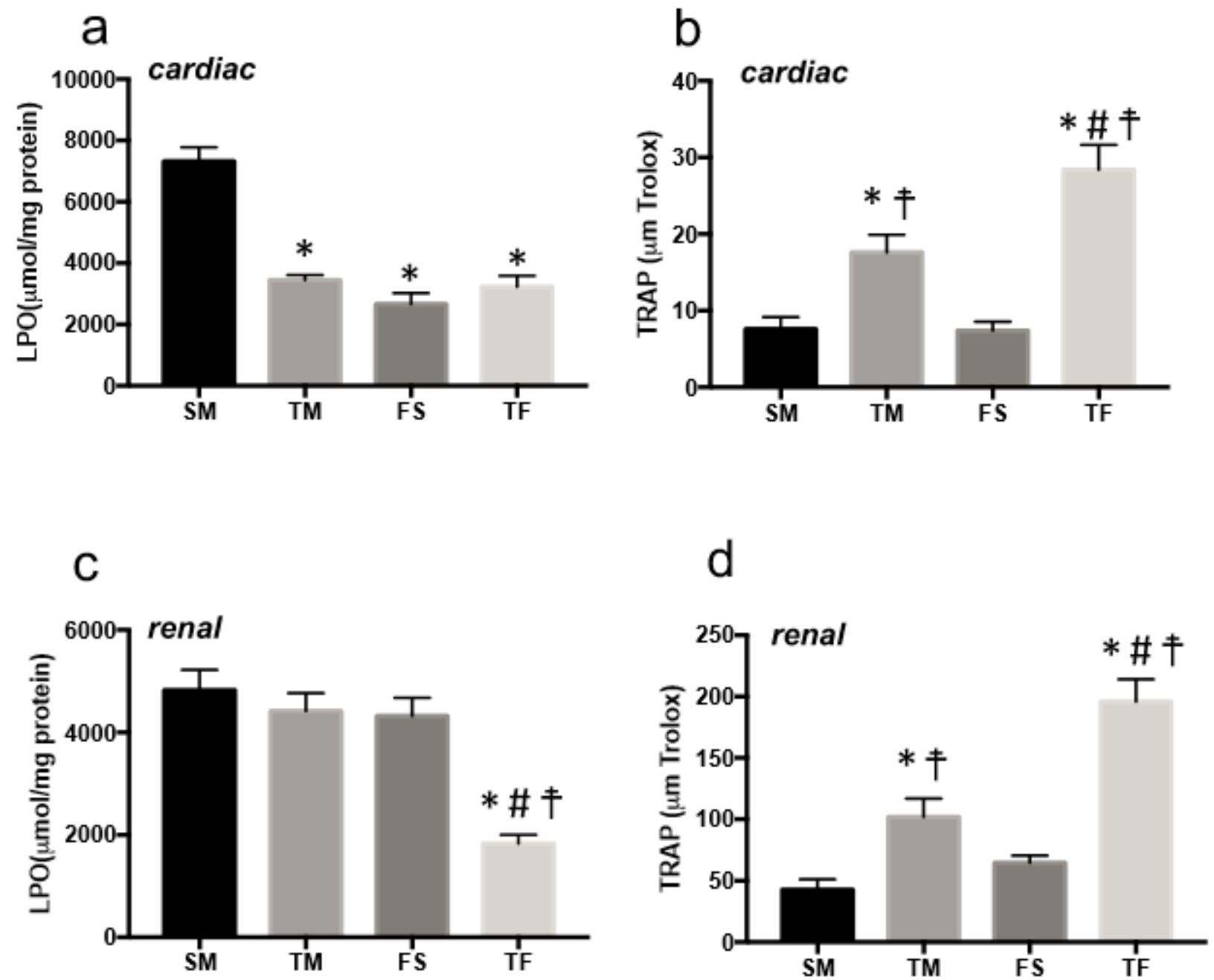

Figure 2

Trained female showed better cardiac and renal oxidative stress profile 
a

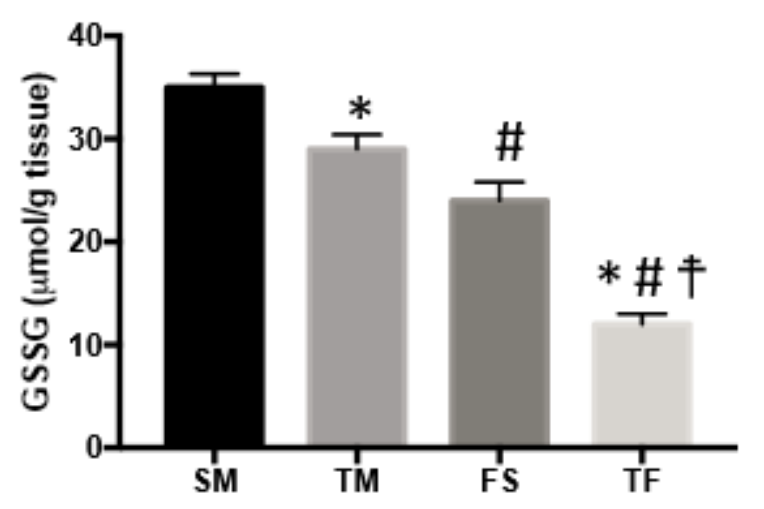

C

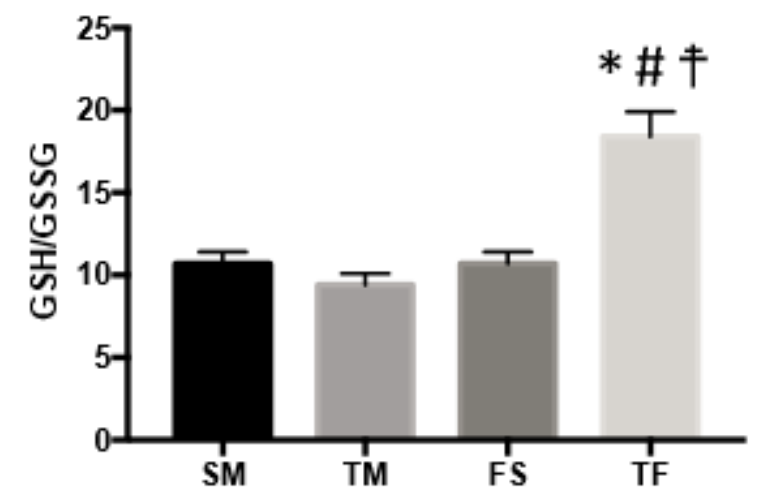

b

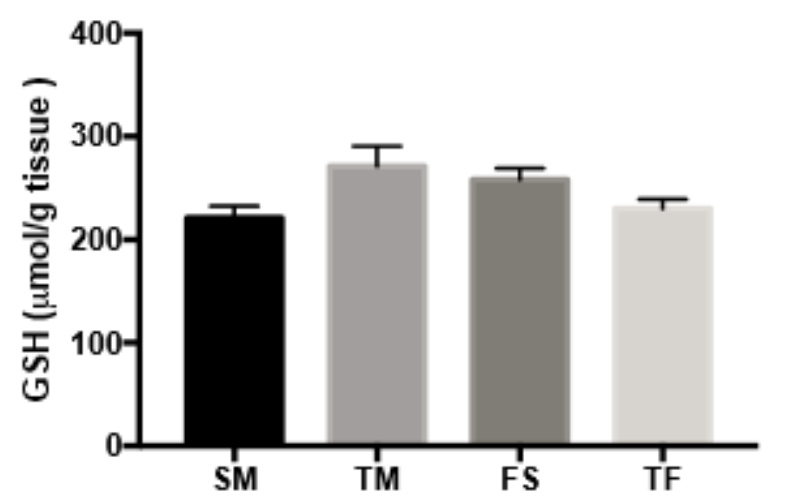

Figure 3

Trained females presented higher antioxidant capacity 

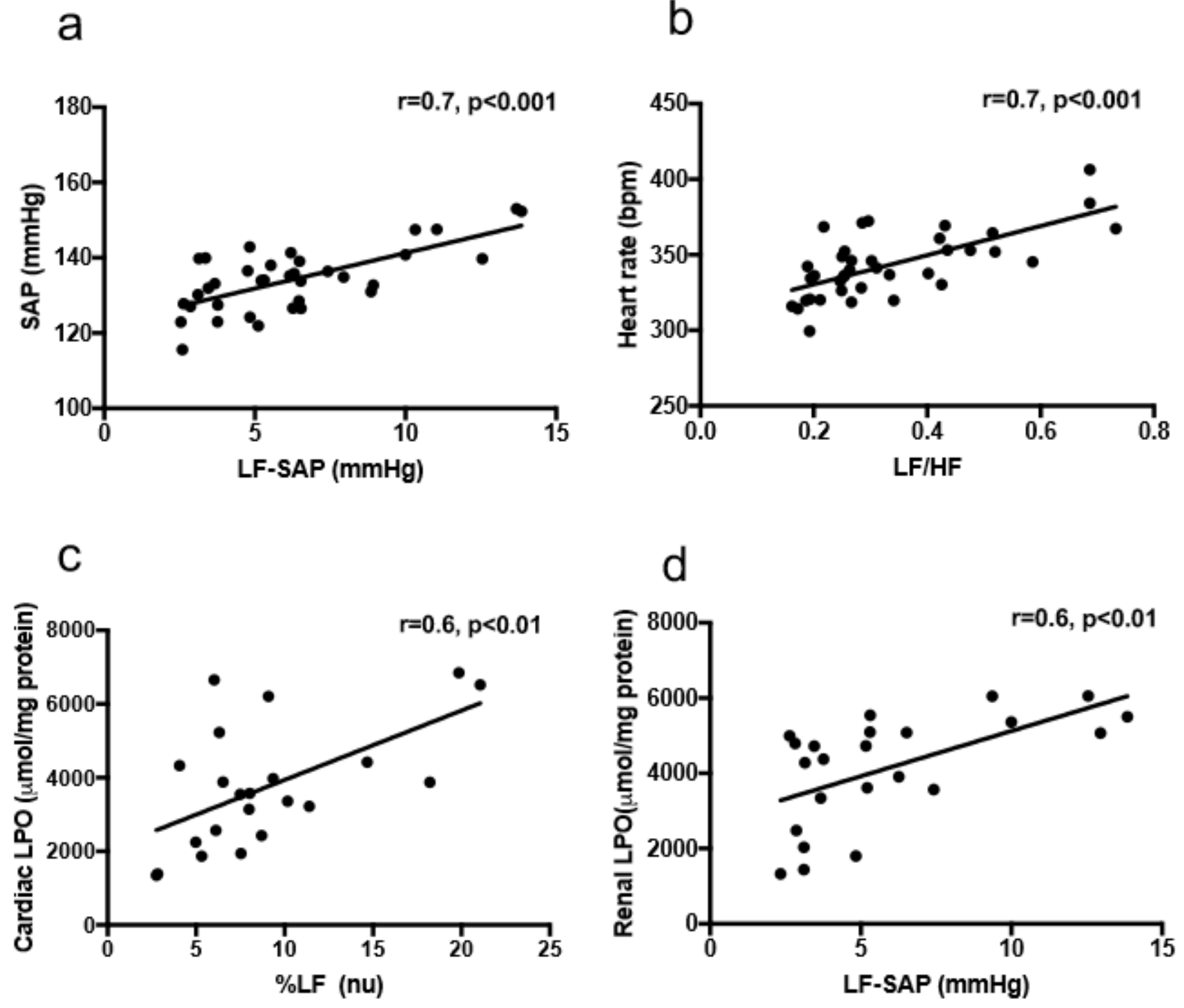

Figure 4

cardiovascular autonomic modulation was correlated with basal hemodynamics and oxidative damage in target organs 